\title{
Integrating a prospective pilot trial and patient-derived xenografts to trace metabolic changes associated with acute myeloid leukemia
}

\author{
Matteo G. Carrabba ${ }^{1 \dagger}$, Laurette Tavel ${ }^{2+}$, Giacomo Oliveira ${ }^{3}$, Alessandra Forcina ${ }^{1}$, Giacomo Quilici ${ }^{2}$, \\ Francesca Nardelli ${ }^{2}$, Cristina Tresoldi ${ }^{4}$, Alessandro Ambrosi ${ }^{5}$, Fabio Ciceri ${ }^{1,6^{*}}$, Massimo Bernardi ${ }^{1}$, Luca Vago ${ }^{1,3+}$ \\ and Giovanna Musco ${ }^{2 \dagger}$
}

\begin{abstract}
Despite the considerable progress in understanding the molecular bases of acute myeloid leukemia (AML), new tools to link disease biology to the unpredictable patient clinical course are still needed. Herein, high-throughput metabolomics, combined with the other "-omics" disciplines, holds promise in identifying disease-specific and clinically relevant features.

In this study, we took advantage of nuclear magnetic resonance (NMR) to trace AML-associated metabolic trajectory employing two complementary strategies. On the one hand, we performed a prospective observational clinical trial to identify metabolic changes associated with blast clearance during the first two cycles of intensive chemotherapy in nine adult patients. On the other hand, to reduce the intrinsic variability associated with human samples and AML genetic heterogeneity, we analyzed the metabolic changes in the plasma of immunocompromised mice upon engraftment of primary human AML blasts.

Combining the two longitudinal approaches, we narrowed our screen to seven common metabolites, for which we observed a mirror-like trajectory in mice and humans, tracing AML progression and remission, respectively. We interpreted this set of metabolites as a dynamic fingerprint of AML evolution.

Overall, these NMR-based metabolomic data, to be consolidated in larger cohorts and integrated in more comprehensive system biology approaches, hold promise for providing valuable and non-redundant information on the systemic effects of leukemia.
\end{abstract}

Keywords: Acute myeloid leukemia, Metabolomics, Patient-derived xenografts, Nuclear magnetic resonance

Re-programming energy metabolism is a hallmark of cancer [1]. Acute myeloid leukemia (AML) permits analysis of the impact of a systemic cancer on the metabolism over time. Oncometabolites have been demonstrated in 5-20\% of AML-harboring mutations in Isocitrate Dehydrogenase (IDH) genes [2, 3], and the metabolic profile of AML cell lines can be employed to

\footnotetext{
* Correspondence: cicerifabio@hsr.it

${ }^{\dagger}$ Equal contributors

${ }^{1}$ Unit of Hematology and Bone Marrow Transplantation, IRCCS San Raffaele Scientific Institute, Via Olgettina 60, 20132 Milan, Italy

${ }^{6}$ University Vita-Salute San Raffaele, Milan, Italy

Full list of author information is available at the end of the article
}

investigate drug treatments [4, 5]. Moreover, previous studies linked AML with perturbation of metabolic pathways including glucose metabolism [6, 7]. Nuclear magnetic resonance (NMR) is a rapid, highly reproducible cost-effective analytical tool to profile metabolic fluctuations, requiring minimal sample manipulation and well suited for automation and high-throughput purposes, thus ideal for untargeted analysis in clinical applications [8-11].

To study the metabolic changes associated with AML in patients, we enrolled nine newly diagnosed patients in a prospective trial (METAM-02 trial, Additional file 1) and monitored the metabolic trajectory of blast clearance during the first two cycles of intensive chemotherapy $(\mathrm{CT})$ 
a

\begin{tabular}{|c|c|c|c|c|c|c|c|c|c|}
\hline UPN\# & Sex, Age & Diagnosis & \%blastsPB & \%blastsBM & Cytogenetics & Molealar Alterations & Hyperleukocytosis & Chemotherapy & Final Outcome \\
\hline 1 & $F, 58$ & denovo AML & 0 & 44 & complex & none & no & $3+7$, FLAGIDA & Dead in CR1 $^{\pi}$ \\
\hline 2 & $F, 68$ & de novo AML & 0 & 22 & normal & CEBPA (single mutated) & no & $3+7$, FLAGIDA & Alive in CR1 \\
\hline 3 & $\mathrm{~F}, 24$ & denovo AML & 87 & 70 & normal & DNMT3A, IDH1, NPM1, FLT3-ITD & yes & ICE, FLAGIDA & Dead in relapse ${ }^{\star x}$ \\
\hline 4 & $M, 42$ & de novo AML & 56 & 40 & $\operatorname{del}(Y), t(8 ; 21)$ & $\mathrm{NT}$ & no & ICE, IC & Alive in CR1 \\
\hline 5 & M, 52 & de novo AML & 36 & 47 & complex & none & no & ICE, MAMACS & Alive in CR1 \\
\hline 6 & $F, 73$ & de novo AML & 59 & 64 & normal & IDH1, NPM1, FLT3-ITD & no & $3+7$, MAMAC & Alive in CRI \\
\hline 7 & $F, 31$ & de novo AML & 14 & n.a. & del(12p) & none & no & ICE, FLAGIDA & Alive in CR1 \\
\hline 8 & $\mathrm{~F}, 52$ & de novo AML & 15 & 84 & complex & $\mathrm{IDH} 2$ & no & ICE, FLAG-IDA & Alve in CRI \\
\hline 9 & $F, 42$ & secondary AML & 4 & 46 & del(11q) & none & no & ICE, MAMACS & Alve in CRI \\
\hline
\end{tabular}

b

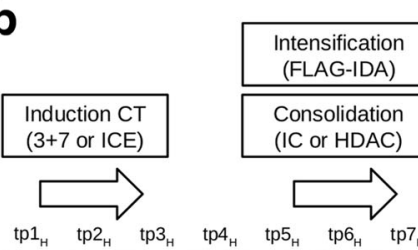

$\begin{array}{lllllllll}\operatorname{tp} 1_{\mathrm{H}} & \operatorname{tp} 2_{\mathrm{H}} & \operatorname{tp} 3_{\mathrm{H}} & \operatorname{tp} 4_{\mathrm{H}} & \mathrm{tp} 5_{\mathrm{H}} & \mathrm{tp} 6_{\mathrm{H}} & \operatorname{tp} 7_{\mathrm{H}} & \operatorname{tp} 8_{\mathrm{H}} & \operatorname{tp} 9_{\mathrm{H}}\end{array}$

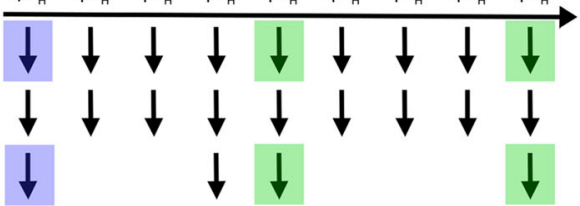

d

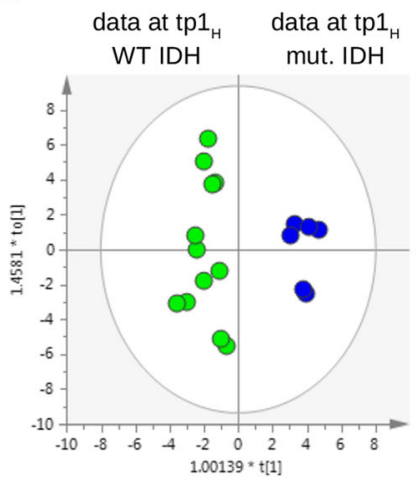

e
C

BM at tp1 BM at $\operatorname{tp} 9_{\mathrm{H}}$

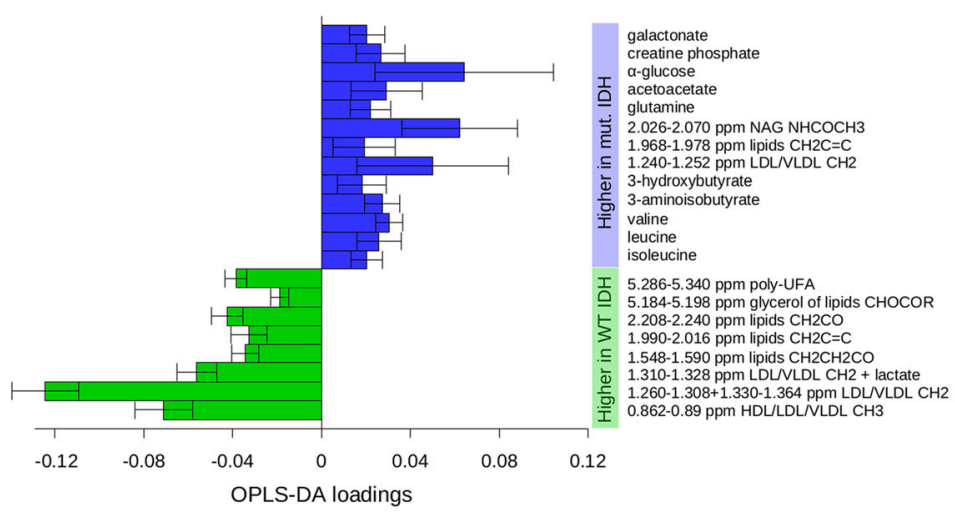

g
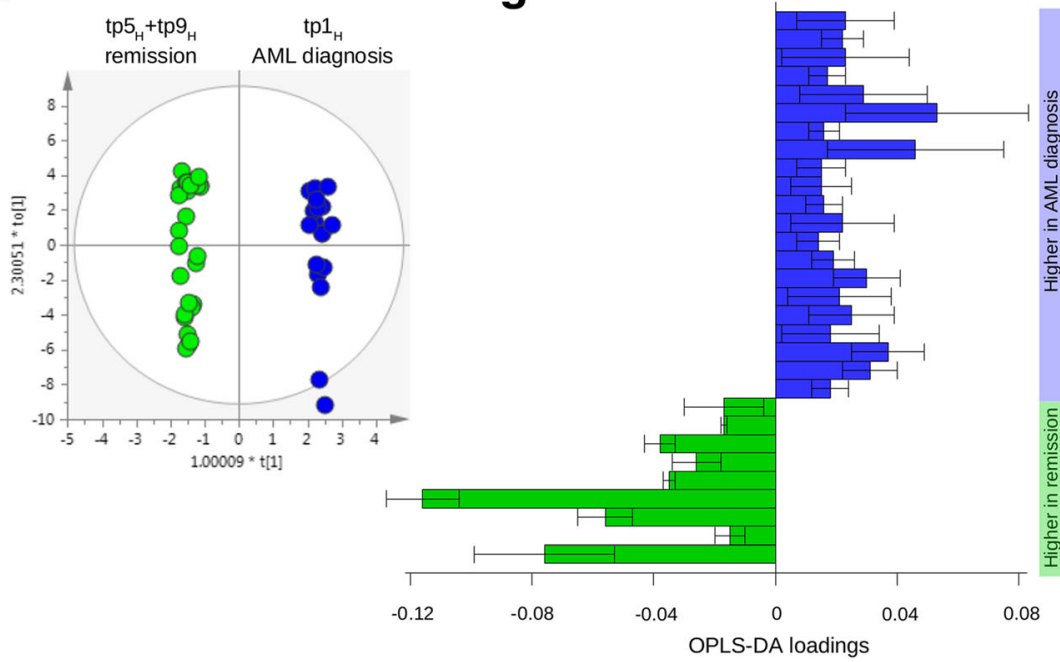

Fig. 1 (See legend on next page.)
PB plasma Urine collection BM plasma collection

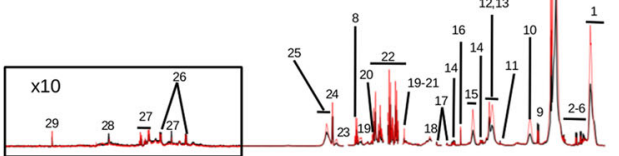

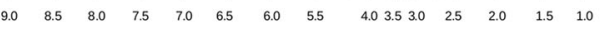
Chemical shifts (ppm) 
(See figure on previous page.)

Fig. 1 Metabolic changes in AML patients undergoing intensive chemotherapy. a Patient characteristics. CR1 first complete remission. *UPN\#1 developed a lethal fungal pneumonia while in remission after the first consolidation CT cycle. **UPN\#3 underwent allogeneic hematopoietic stem cell transplantation in CR1 and subsequently relapsed. $\mathbf{b}$ Outline of the study design. c Superposition of representative ${ }^{1} \mathrm{H}$ Carr-Purcell-MeiboomGill (CPMG) spectra of BM at tp1 $1_{H}$ (black line) and $\mathrm{tp}_{\mathrm{H}}$ (red line) acquired at $37^{\circ} \mathrm{C}$ on a Bruker Avance $600-\mathrm{MHz}$ spectrometer. The ${ }^{1} \mathrm{H} C \mathrm{CPMG}$ NMR experiment is based on a pulse sequence that strongly reduces the NMR signals deriving from large molecules; herewith, molecules with high molecular weight are essentially invisible in the ${ }^{1} \mathrm{H}$ spectrum, thus facilitating spectra interpretation and small molecule identification in the presence of large proteins and lipoproteins. Peaks correspond to the different metabolites: 1-high-, low-, and very low-density lipoproteins (HDL, LDL, VLDL) CH3; 2-isoleucine; 3-leucine; 4-valine; 5-3-aminoisobutyrate; 6-3-hydroxybutyrate; 7-LDLNLDL CH2; 8-lactate; 9-alanine; 10-lipids $\mathrm{CH} 2 \mathrm{CH} 2 \mathrm{CO}$; 11-acetate; 12-lipids $\mathrm{CH} 2 \mathrm{C}=\mathrm{C} ; 13$ - N-acetyl-glycoproteins (NAG) NHCOCH3; 14-glutamine; 15-lipids $\mathrm{CH} 2 \mathrm{CO}$; 16-acetoacetate; 17—citrate; 18 -lipids $\mathrm{C}=\mathrm{CCH} 2 \mathrm{C}=\mathrm{C}$; 19-creatinine; 20 - creatine; 21—creatine phosphate; 22-glucose; 23-glycerol of lipids CHOCOR; 24—a glucose; 25-poly-unsaturated fatty acids (UFA); 26-tyrosine; 27-phenylalanine; 28-histidine; 29-formate. d OPLS-DA score plot for pooled PB and BM samples collected at diagnosis and typing positive (blue circles; $n=6$ ) or negative (green circles; $n=12$ ) for missense mutations in the IDH1/2 genes. OPLS-DA with $N=18$, CV ANOVA $p=0.059, R^{2}=0.93$, and $Q^{2}=0.615$. The area under the curve (AUC) of the ROC analysis was 0.86 ( $\left.p<0.001\right)$. e Metabolites discriminating AML patients with or without IDH gene mutations. Loadings indicate how much the variables, i.e., the metabolites, contribute to the model. Shown are loadings with jack-knifed confidence interval. The metabolites significantly contributing to the model were selected based on variable importance in projection $>1$ and jack-knifed confidence interval of loadings not crossing the zero line. Positive loading values (blue bars) indicate the metabolites increased in patients with mutated IDH, while negative values (green bars) are associated with increased levels in patients with wild-type IDH. Note that the concentration levels of the classical IDH mutation oncometabolite (R)-2-hydroxyglutarate were below the NMR detection limit and that because of the overlap of the resonances associated to $\mathrm{CH} 2$ groups of high-, low- and very low-density lipoproteins (HDL, LDL, VLDL) and the $\mathrm{CH} 2 \mathrm{C}=\mathrm{C}$ groups of different lipid molecules it was not possible to establish their specific contribution to the model. $\mathbf{f}$ OPLS-DA score plot for pooled $\mathrm{PB}$ and $\mathrm{BM}$ samples of patients at diagnosis ( $\mathrm{tp} 1_{\mathrm{H}}$, blue circles; $n=18$ ) vs remission after chemotherapy (pooling samples after induction, tp5 $5_{H}$, and after first consolidation, $\mathrm{tp}_{\mathrm{H}}$, green circles; $n=29$ ). OPLS-DA model with $N=47, \mathrm{CV}$-ANOVA $p=0.006, R^{2}=0.99$, and $Q^{2}=0.671$. The AUC of the ROC analysis was $0.73(p<0.001)$. g Metabolites discriminating AML patients at diagnosis and in remission after chemotherapy. Positive loading values (blue bars) indicate the metabolites increased in AML patients at $\mathrm{tp} 1_{\mathrm{H}}$, while negative values (green bars) are associated with increased metabolite levels at tp5 $5_{H}+\mathrm{tp} 9_{\mathrm{H}}$

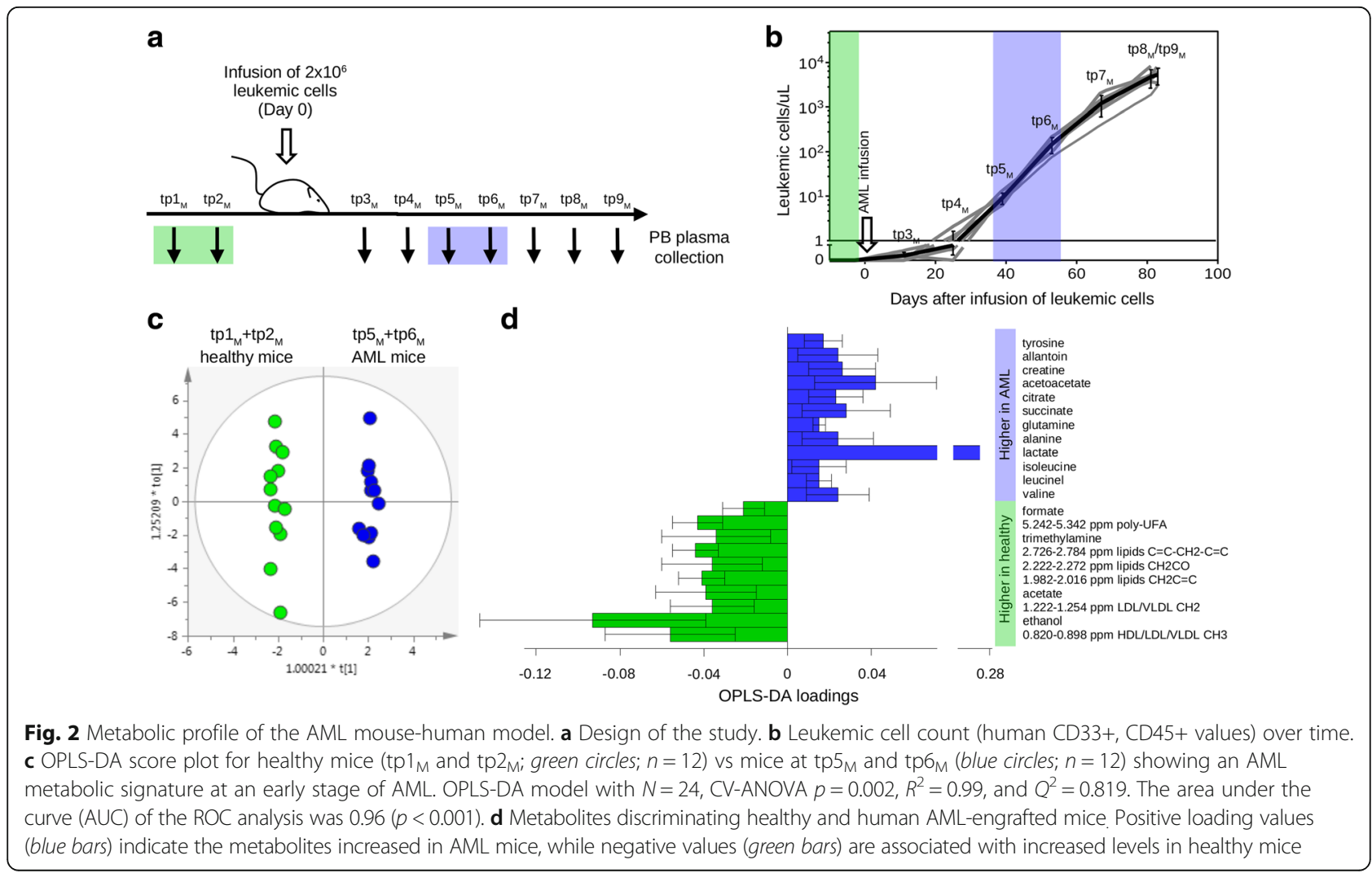


(Fig. 1a). Peripheral blood (PB), bone marrow (BM), and urine samples were collected prior, during, and after induction and consolidation $\mathrm{CT}$, resulting in nine time points $\left(\mathrm{tp}_{\mathrm{H}}\right)$ (Fig. $\left.1 \mathrm{~b}\right)$. At the final time point $\left(\mathrm{tp} 9_{\mathrm{H}}\right)$, all patients had achieved complete remission.

Seventy-four PB, 33 BM, and 75 urine samples were analyzed by NMR spectroscopy (Fig. 1c and Additional file 1). First, based on results from unsupervised statistical inspection, we decided to pool BM samples with the respective $\mathrm{PB}$ and observed that despite the small number of cases, supervised orthogonal partial least squares-discriminant analysis (OPLS-DA) allowed clustering of the samples at diagnosis $\left(\mathrm{tp} 1_{\mathrm{H}}\right)$ according to the presence or absence of mutations in IDH genes (Fig. 1d, e), supporting the notion that these mutations have an overall impact on the metabolome $[2,3]$. Moreover, OPLS-DA discriminated the metabolic differences between patients at disease diagnosis and those in remission $\left(\mathrm{tp} 5_{\mathrm{H}}\right.$ and $\mathrm{tp} 9_{\mathrm{H}}$ ), highlighting 30 metabolites significantly contributing to the model (Fig. 1f, g).

To pinpoint the most relevant ones for AML biology, we integrated our study with a mouse-human AML model, allowing us to follow the metabolic changes associated with disease progression. Six littermate NOD/ SCID $\gamma$-chain-null (NSG) mice were infused with $2 \times 10^{6}$ primary AML blasts (from patient \#3). PB samples were collected over time from the same animals before and after AML infusion for a total of nine time points ( $\left.\mathrm{tp}_{\mathrm{M}}\right)$ (Fig. 2a). AML became detectable (blast count $>1$ cell/ $\mu \mathrm{l})$ in the $\mathrm{PB}$ at $\operatorname{tp} 5_{\mathrm{M}}$ for all infused mice; thereafter, an exponential growth started (Fig. 2b). We thus compared samples before AML infusion ( $\mathrm{tp} 1_{\mathrm{M}}$ and $\mathrm{tp} 2_{\mathrm{M}}$ ) and in the exponential phase of disease (tp $5_{M}$ and $t p 6_{M}$ ). Possibly due to the lower number of variables present in the mouse model, OPLS-DA (Fig. 2c) pinpointed only 22 metabolites accounting for AML progression, mainly involved in pathways related to energy and fatty acid metabolism (Fig. 2d). The increased lactate level in the plasma is a typical hallmark of cancer, characterized by an aerobic glycolytic shift ("Warburg effect") [1, 12]. Lower plasma levels of cholesterol, lipids, and unsaturated fatty acids were found in AML mice than in healthy controls, in agreement with previous reports $[6,13,14]$, highlighting an involvement of fatty acid metabolisms in

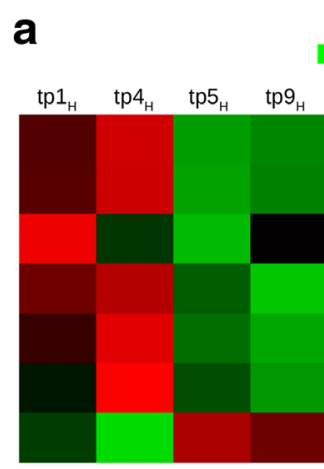

Human BM
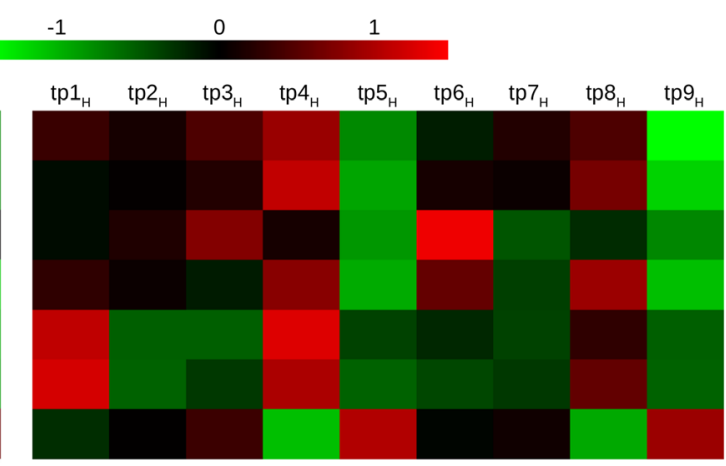

Human PB
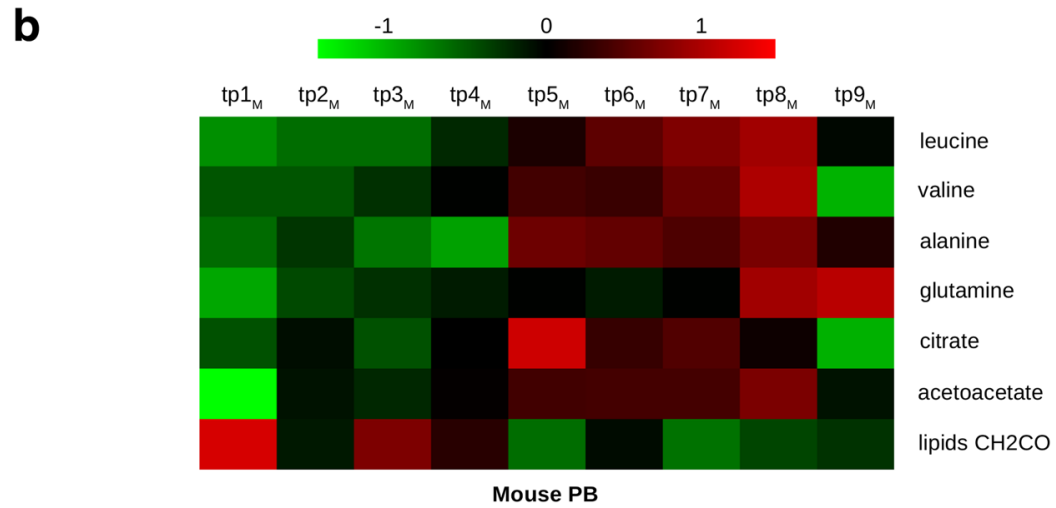

b

Fig. 3 Heat maps of human or mouse plasma tracing the trajectory of the seven metabolites associated with AML evolution in both the patients and mice. a Heat maps of human BM and PB tracing the trajectory of the seven metabolites (averaged normalized metabolite area) associated with AML evolution in both the mice and patients. $\mathbf{b}$ Heat map of mouse PB depicting the trajectory of the seven metabolites (averaged normalized metabolite area of the six mice) associated with AML evolution in both the mice and patients. Note that leucine, valine, alanine, citrate, and acetoacetate increased upon AML progression until tp $8_{M}$ and then decreased at tp $9_{M}$. This reverse effect supported our hypothesis about a metabolic change due to overall systemic failure at a late stage of disease and not to a specific effect of AML 
AML, possibly related to the demand for lipids and cholesterol in tumor proliferation. Also, the increase of glutamine in AML mice is consistent with cancer since glutamine is a major carbon and nitrogen source for tumor cell proliferation [15].

Combining the two longitudinal approaches to trace AML in patients and mice, we narrowed our screen to seven metabolites representative of AML tumor burden: leucine, valine, alanine, glutamine, citrate, acetoacetate, and lipids $\mathrm{CH} 2 \mathrm{CO}$. We interpreted this set of metabolites as a dynamic fingerprint of AML evolution that could be followed in a mirror-like trajectory in both studies (Fig. 3).

Collectively, our results highlight how NMR-based metabolomics might provide valuable and non-redundant information on the systemic effects of leukemia, to be consolidated in larger cohorts, and integrated in more comprehensive system biology approaches. Although the limited number of cases investigated in this study does not allow a meaningful comparison to previous reports [6, 7], the technical robustness and reproducibility of NMR, together with the minimal sample manipulations and invasiveness of this technique, appear well suited to perform longitudinal studies in clinical settings. Moreover, we first provide experimental evidence on how patient-derived xenografts can be integrated into metabolomic studies, complementing and narrowing the screening of clinically relevant metabolites.

\section{Additional file}

Additional file 1: Supplementary Methods, Results and Figures. (PDF 966 kb)

\section{Acknowledgements}

The authors would like to thank Stefania Trinca and all the San Raffaele URC SCP (Clinical Research Office) staff. Laurette Tavel was supported by a fellowship from Fondazione Veronesi, Giacomo Oliveira was supported by a fellowship from Fondazione Matterelli sponsored by the Associazione Italiana Leucemie.

\section{Funding}

This work was supported by a generous donation from the Comitato Ricerca Contro la Leucemia (CRCL) and by grants provided from the Italian Ministry of Health (RF-2011-02351998, RF-2013-02354880 and TRANSCAN HLALOSS), by the Associazione Italiana per la Ricerca sul Cancro (Start-Up Grant \#14162 and Investigator Grant\#17468), by the Conquer Cancer Foundation (2014 Young Investigator Award), and by the DKMS Mechtild Harf Foundation.

\section{Availability of data and materials}

Not applicable. All data and materials supporting the conclusions of this article are included within the article.

\section{Competing interests}

The authors declare that they have no competing interests.

\section{Consent for publication}

Not applicable.

\section{Ethics approval and consent to participate}

The METAM02 prospective clinical trial has been approved by the San Raffaele Ethic Committee, and all patients signed informed consent upon enrollment. All animal experiments were performed upon approval from the San Raffaele Institutional Animal Care and Use Committee (IACUC).

\section{Author details}

${ }^{1}$ Unit of Hematology and Bone Marrow Transplantation, IRCCS San Raffaele Scientific Institute, Via Olgettina 60, 20132 Milan, Italy. ${ }^{2}$ Biomolecular Nuclear Magnetic Resonance Unit, IRCCS San Raffaele Scientific Institute, Milan, Italy. ${ }^{3}$ Unit of Immunogenetics, Leukemia Genomics and Immunobiology, IRCCS San Raffaele Scientific Institute, Milan, Italy. ${ }^{4}$ Molecular Hematology Laboratory, IRCCS San Raffaele Scientific Institute, Milan, Italy. ${ }^{5}$ Center for Statistics in Biomedical Sciences, University Vita-Salute San Raffaele, Milan, Italy. ${ }^{6}$ University Vita-Salute San Raffaele, Milan, Italy.

Received: 26 August 2016 Accepted: 18 October 2016

Published online: 28 October 2016

\section{References}

1. Hanahan D, Weinberg RA. Hallmarks of cancer: the next generation. Cell. 2011;144:646-74.

2. Losman JA, Looper RE, Koivunen P, Lee S, Schneider RK, McMahon C, et al. (R)-2-hydroxyglutarate is sufficient to promote leukemogenesis and its effects are reversible. Science. 2013;339:1621-5.

3. McKenney AS, Levine RL. Isocitrate dehydrogenase mutations in leukemia. J Clin Invest. 2013;123:3672-7.

4. Tiziani S, Lodi A, Khanim FL, Viant MR, Bunce CM, Gunther UL. Metabolomic profiling of drug responses in acute myeloid leukaemia cell lines. PLoS One. 2009;: 4 :e4251.

5. Lodi A, Tiziani S, Khanim FL, Drayson MT, Gunther UL, Bunce CM, et al. Hypoxia triggers major metabolic changes in AML cells without altering indomethacininduced TCA cycle deregulation. ACS Chem Biol. 2011;6:169-75.

6. Wang Y, Zhang L, Chen WL, Wang JH, Li N, Li JM, et al. Rapid diagnosis and prognosis of de novo acute myeloid leukemia by serum metabonomic analysis. J Proteome Res. 2013;12:4393-401.

7. Chen WL, Wang JH, Zhao AH, Xu X, Wang YH, Chen TL, et al. A distinct glucose metabolism signature of acute myeloid leukemia with prognostic value. Blood. 2014;124:1645-54.

8. Emwas AH, Luchinat C, Turano P, Tenori L, Roy R, Salek RM, et al. Standardizing the experimental conditions for using urine in NMR-based metabolomic studies with a particular focus on diagnostic studies: a review. Metabolomics. 2015;11:872-94.

9. Nagana Gowda GA, Raftery D. Can NMR solve some significant challenges in metabolomics? J Magn Reson. 2015;260:144-60.

10. Alonso A, Marsal S, Julia A. Analytical methods in untargeted metabolomics: state of the art in 2015. Front Bioeng Biotechnol. 2015;3:23.

11. Nicholson JK, Holmes E, Kinross JM, Darzi AW, Takats Z, Lindon JC. Metabolic phenotyping in clinical and surgical environments. Nature. 2012; 491:384-92

12. Warburg O. On the origin of cancer cells. Science. 1956;123:309-14.

13. Tiziani S, Kang Y, Harjanto R, Axelrod J, Piermarocchi C, Roberts W, et al. Metabolomics of the tumor microenvironment in pediatric acute lymphoblastic leukemia. PLoS One. 2013;8:e82859.

14. Beloribi-Djefaflia S, Vasseur S, Guillaumond F. Lipid metabolic reprogramming in cancer cells. Oncogenesis. 2016;5:e189.

15. Souba WW. Glutamine and cancer. Ann Surg. 1993;218:715-28.

\section{Authors' contributions}

MGC, FC, LV, and GM designed the study. LT, GQ, FN, and GM performed and analyzed the NMR experiments. GO and LV designed, performed, and analyzed the mouse experiments. MGC, AF, FC, and MB contributed to the patient clinical care and data collection. CT supervised the sample collection and banking. AA supervised statistical analyses. MGC, LT, GO, FC, LV, and GM wrote the manuscript. All authors read and approved the final manuscript. 